\title{
EL RECORRIDO DE LAS METODOLOGÍAS DE PRINCIPIOS DEL SIGLO XX EN LA ENSEÑANZA DE LA MÚSICA EN ESPAÑA: APROXIMACIÓN BIBLIOGRÁFICA E INTERPRETATIVA.
}

\author{
THE ROUTE OF METHODOLOGIES OF THE BEGINNING OF THE \\ TWENTIETH CENTURY IN THE TEACHING OF MUSIC IN SPAIN: \\ BIBLIOGRAPHICAL AND INTERPRETATIVE APPROACH
}

\author{
Juan Carlos Montoya Rubio \\ Universidad de Murcia \\ jcmontoyarubio@gmail.com \\ ORCID iD: https://orcid.org/0000-0002-1697-0023
}

\begin{abstract}
Resumen
En las etapas obligatorias, las metodologías de enseñanza musical en España poseen unas características muy definidas, basadas en la observación y adaptación de algunos métodos considerados ya clásicos (especialmente las propuestas de Orff, Kodály o Jaques-Dalcroze). Ello ha generado unas praxis concretas y una dotación material encaminada a cubrir unas expectativas y necesidades concretas. El modelo educativo musical español ha evolucionado de acuerdo con estos parámetros. Por ello, es esencial rastrear los primeros textos que adoptaron estos métodos y analizar el modo en que la bibliografía posterior los ha abordado, desarrollado y plasmado. Todo ello, unido a las inflexiones legislativas, servirá para encontrar claves interpretativas a los modelos pedagógicos que han llegado hasta nuestros días.
\end{abstract}

\section{Palabras clave}

Educación Musical, Metodologías Musicales, OrffSchulwerk, Método Dalcroze, Método Kodály, Bibliografía, Cursos de Formación Musical.

\section{INTRODUCCIÓN}

Los modelos musicales que se desarrollan en las aulas de la enseñanza obligatoria de España tienen un porqué no siempre reflexionado. Ello implica la aceptación sin más de determinados criterios a la hora de abordar los procesos educativos, que van desde los propios contenidos que se ofrecen

\begin{abstract}
In the obligatory stages, the methodologies of musical education in Spain have clear characteristics, based on the observation and adaptation of some classic methods (especially the proposals of Orff, Kodály or Jaques-Dalcroze). This has generated concrete procedures and school equipment to meet the expectations and needs of these methods. The Spanish musical educational model has evolved according to these parameters. Therefore, it is essential to trawl through the files that adopted these methods and to analyze the way in which the later bibliography has approached, developed and shaped them. All this, together with the legislative changes, will serve to find interpretive keys to the pedagogical models of today.
\end{abstract}

\section{Keywords}

Musical Education, Musical Methodologies, Orff-Schulwerk, Dalcroze Method, Kodály Method, Bibliography, Music Training Courses.

a los alumnos hasta la dotación instrumental de las aulas, entre otras contingencias. El objetivo principal de este texto es el de mostrar, a través de un filtrado de bibliografía relevante, cuáles fueron las claves en la fijación de las metodologías más recurrentes en nuestro contexto lo cual, finalmente, nos conducirá a razonamientos acerca de la gestación de la praxis pedagógico-musical actual. De acuerdo con este 
planteamiento, se huirá del detallado de enfoques didácticos para preocuparnos, desde un prisma más histórico y relacional, de los motivos y controversias que surgieron desde que los modelos de Jaques-Dalcroze, Orff o Kodály, por citar a los más recurrentes, empezaran a tomarse seriamente en consideración para implementarse en nuestro sistema educativo obligatorio. Entendemos pues que las herramientas de estudio musicológico son esenciales en un acercamiento de este perfil, ya que el carácter explicativo que se persigue para el campo de la educación musical, aun no siendo el principal centro de interés de la musicología, desde Guido Adler -si no se quiere filosofar sobre aportaciones anteriores en nuestra ciencia- forma parte de ella y no puede ser soslayado.

Como se ha apuntado, la práctica educativa musical en los centros de enseñanza obligatoria de la España del presente es deudora, en gran medida, de los planteamientos didácticos que parten de la primera mitad del siglo XX. A pesar de la distancia temporal y de las actualizaciones, determinados estudios nos invitan a pensar que las metodologías utilizadas a día de hoy, o aquellas que los docentes en activo conocen con más profusión, son las que responden al ideario de autores que forjaron sus axiomas en el resto de Europa. Al margen de las disquisiciones que consideren la pertinencia de su uso en la sociedad del presente, nos interesa especialmente analizar, de manera diacrónica, los porqués de esta instalación a partir de las primeras aportaciones de los autores en cuestión que fueron adaptadas o traducidas al castellano. Aunque nuestra principal pretensión sea la de atender al contexto español, es necesario precisar que algunas de estas obras señeras fueron conocidas a partir de su publicación en Sudamérica (especialmente en Argentina), por lo que esas referencias también nos serán válidas. De hecho es común remitirse todavía hoy a estas ediciones ante la ausencia de adaptaciones impresas en España. Con ello, trataremos de arrojar luz a la mixtura metodológica actual y al abanico de posibilidades que se han abierto, especialmente, desde finales del siglo XX, cuando a pesar de los altibajos atestiguados en la entrada de la siguiente centuria, la música comenzó a ser tratada con cierto rigor en las aulas de educación musical.

Para el propósito que nos marcamos es preceptivo, en primer lugar, realizar un breve análisis de las obras de referencia, teniendo en cuenta los modelos que las vieron surgir. No pretendemos detallar cada una de las reseñas aparecidas, sino más bien rescatar aquellas que sirven para trazar una línea de continuidad en la transmisión metodológica. En este sentido, se estructuran los contenidos señalando aquellos aspectos que serán retomados por obras que beban de esas fuentes, de forma que se pueda cotejar la presencia de sus elementos definitorios y, además, se hagan patentes las aportaciones novedosas que presenten. A continuación, lle- gados a esta bibliografía secundaria, se enfatiza la necesidad de establecer paralelismos entre esas obras y la labor de difusión que se llevó a cabo merced a los diversos simposia y cursos de especialización conducidos bien por los autores que encabezaban esos métodos bien por sus seguidores, siendo este aspecto de vital importancia para poder baremar hasta qué punto se ha llegado a un desarrollo más o menos exitoso del modelo pedagógico-musical en cuestión fuera de sus fronteras. Finalmente, el tercero de los apartados se aproximará al modo en que, una vez recibidos y conocidos sus postulados, se les da forma dentro del contexto de una educación musical, la española, que carecía de una estructura mínimamente sólida hasta la década de 1990, cuando se constituye de manera más firme la figura del especialista en educación musical y se ha de formar dicho perfil desde las universidades, por supuesto, con los criterios metodológicos venidos de las primeras décadas del siglo.

\section{DEL CAMBIO METODOLÓGICO EN LA PEDAGOGÍA MUSICAL MÁS ALLÁ DE LOS PIRINEOS}

En la década de los cincuenta del siglo pasado (si bien la traducción es posterior) Rudolf Schoch comentaba que

el enriquecimiento del sentido para los sonidos, obtenido en virtud de las sugestiones de Carl Orff y Hans Bergese, mediante el empleo de instrumentos de percusión y de sus acompañamientos modernos, es de la mayor importancia $[\ldots]$

La música escolar hará bien en hacer suyas, en el interés de la formación del ser humano, las ideas fundamentales de Carl Orff, y entonces será realmente capaz de entusiasmar y modelar a la juventud ${ }^{1}$.

La declaración de intenciones anterior se escribe poco más de veinte años después de que Orff empezase a publicar sus planteamientos pedagógicos y, a día de hoy, bien entrado el siglo XXI, hemos de señalar que el deseo expresado no quedó en saco roto, dado que en la actualidad encontramos constantemente ediciones que versan sobre la obra pedagógica de Orff, asociaciones que se entregan a su recuerdo, desarrollo y evolución y que, al igual que con otros referentes como Kodály o Jaques-Dalcroze, publican revistas especializadas con diversas praxis que se nutren del mismo tronco común o que organizan cursos que, a lo largo y ancho del mundo, tienen como argumento nodal la irradiación de sus criterios procedimentales. A este último respecto, la

1 SCHOCH (1964): 8-9; (1964): 18. 
primera reflexión que hemos de poner de manifiesto es delimitar quiénes son esos autores, porque no todos han tenido el mismo peso en el proceso de fijación que se ha llevado a cabo en el territorio español durante la segunda mitad del siglo pasado.

En un estudio que trataba de medir la incidencia de las metodologías en las aulas, $\mathrm{Oriol}^{2}$ establecía que, por este orden, Carl Orff, Zoltán Kodály y Emile Jaques-Dalcroze (seguido el último a una pequeña distancia por Edgar Willems) eran los pedagogos mejor considerados en nuestras aulas. Cierto es que la muestra del estudio no era lo suficientemente grande como para asegurar que no se pudiera esconder algún sesgo en torno a los resultados, pero no es menos cierto que si nos entrevistamos con docentes en activo y consultamos sobre la orientación que han recibido en las universidades, sobre las metodologías que vehiculan sus clases de manera habitual y sobre el tipo de material (instrumental) con que están dotadas las aulas de educación musical, es más que posible que sean los tres autores señalados por Oriol los que aparezcan con mayor frecuencia. Obviamente no puede ser casual. En cierto modo, esta situación guarda similitudes con la expuesta en su día por Bruno $\mathrm{Nettl}^{3}$ cuando llamaba la atención sobre lo simbólico de las triadas de grandes genios de la música culta, a partir de cuyos nombres encumbrados se elaboraban visiones míticas que ponían en valor sus músicas por encima de otras. En el caso del estudio de los métodos pedagógicos, parece claro a la luz de lo comentado con anterioridad que la triada Orff - Kodály - Dalcroze gozaría de un puesto privilegiado en el imaginario de los docentes mientras que otros pedagogos (con Willems a la cabeza junto con, a saber, Martenot o Ward y otros más actuales como Wuytack, por ejemplo) ocuparían una segunda línea en el panteón simbólico del que nos hablara Nettl. Al margen del potencial de esa terna de métodos y autores es evidente que la correa transmisora de los mismos desde su gestación hasta su posición actual es debida a unas contingencias muy concretas que tienen que ver con sus mecanismos de difusión, pero también con las posibilidades de integración dentro del modelo educativo de acogida, en este caso el español, huérfano de docentes de música en los niveles elementales prácticamente hasta la década de 1990.

En España, la obra a la que suele recurrirse para hablar de la recepción de Orff es el pequeño trabajo (poco voluminoso pero de inmenso valor simbólico y efectivo) de Montserrat Sanuy y Luciano González Sarmiento 4 , tardío sin duda en cuanto a aparición (no tanto por los seis años de desfase con la obra de referencia sino por la ausencia de otras versiones anteriores dentro de España), aspecto tan común en nuestra historia musical que casi no resulta extremadamente extraño. Sumamente clarificador, con un perfil muy didáctico y comprensible, el volumen aborda las líneas maestras del método atendiendo, de este modo, al desarrollo vocal a través de elementales intervalos y escalas, las cuales engarzan con el uso instrumental en el prototípico orden secuencial de percusiones corporales, pequeña percusión de altura indeterminada e instrumental de láminas. Finalmente, la adhesión de la flauta dulce nos conduce a los acompañamientos e improvisaciones. Con todo, la parte inicial de este texto es, tal vez, una de las más influyentes en las prácticas que nos han sido legadas a la actualidad. En ella se desarrolla el elemento rítmico a partir de la asociación de la notación musical con sílabas concretas; de monosílabos se pasa a bisílabos y así, sucesivamente, se van cubriendo las necesidades rítmico-musicales a partir de la palabra, hallándose correspondencias entre tipos de compases y expresiones orales; la sustitución de voces por percusiones rítmico-corporales genera esquemas que dan lugar a polirritmias que van ganando en complejidad con la combinación constante que realiza para, finalmente, introducir las últimas dificultades, producto final del recorrido pedagógico realizado.

La obra de Sanuy y González Sarmiento, completada con el conjunto de rimas, trabalenguas y demás piezas instrumentales que ejemplificaban los modelos didácticos desde el folklore propio ${ }^{5}$, sirvió para abrir las miras pero dejaba, respecto a la metodología de Orff, un inmenso hueco todavía, el que nos conducía de manera profusa y sistemática a casos de aplicaciones instrumentales a varias voces (respondiendo así al material que el propio Orff publicara conjuntamente con Gunild Keetman ${ }^{6}$ ). En ese punto se revalorizan las adaptaciones venidas de Argentina, que vieron la luz poco antes en la obra de Guillermo Graetzer, a la sazón artífice de la adaptación castellana para América Latina del Orff-Schulwerk quien, además de mantener las palabras introductorias del original, añadió para su edición algunas explicaciones que nos sirven para interpretar su manera de proceder:

Se comprenderá fácilmente que una obra de estas características requería una tarea de adaptación mucho más compleja que una simple traducción: era necesario encontrar canciones y rimas análogas a las empleadas por Carl Orff, lo que a su vez exigía una remodelación del material melódico ${ }^{7}$.

\author{
5 SANUY, GONZÁLEZ SARMIENTO (1969b). \\ 6 ORFF, KEETMAN (1952). \\ 7 GRAETZER (1961): vol. 1, s. p.
}


Graetzer, al igual que sucedió con las traducciones a otros idiomas, se encargó de poner en valor la obra de Orff en una adaptación ya clásica, tratando de vincular, en cierto modo, los postulados del autor a la lengua española. La persona que actuó como colaborador (y traductor) de algunos textos para la versión de Graetzer ${ }^{8}$ fue Antonio Yepes. Este autor ejemplifica el entramado de interrelaciones que se establecía entre los pedagogos preocupados por iluminar las obras de los métodos que revolucionaban buena parte de Europa $^{9}$, ya que él, a su vez, firma uno de los textos de referencia en castellano sobre el canto coral desde la perspectiva de Kodály, basándose en sus principios básicos ${ }^{10}$. La importancia de esta última aportación, curiosamente dividida -de manera incluso física- en dos partes (láminas y ejercicios frente a la explicación metodológica) radica en que no sólo plantea los procedimientos tal como los concibió Kodály, sino que además presenta adaptaciones al lector en castellano. Este hecho hace accesibles algunos cantos y sirve para ejemplificar sobre temas populares del lugar con lo que, por consiguiente, torna reconocibles las melodías.

Siguiendo con Zoltán Kodály, otro hito reseñable será la traducción de Oriol Martorell y Marga García (con revisión de Montserrat Coll), la cual sirvió para estampar la firma del compositor y pedagogo húngaro a través del texto de Erzsébet Szönzy ${ }^{11}$. Cualquiera que haya tenido la necesidad de buscar una referencia para explicar en qué consistieron en su origen sus tratados se ha topado, de uno u otro modo, con la obra a la que nos referimos la cual, como otras tantas venidas de Hungría, se gestaron por el interés en ver promocionado desde su país (seña de identidad de la propia editorial Corvina) una obra con garantías de éxito. El texto, trufado de principios de intenciones con referencias a los cursos y conferencias impartidas por Kodály o por gráficos ejemplos de aplicación pedagógica, son un referente todavía hoy. Y lo son porque cumplieron la función de fijación de los elementos centrales de la metodología, dejando el cuño de la autoría en un periodo de concomitancias varias. En este sentido, es curioso observar cómo durante el tiempo de alumbramiento de los propios métodos existían terrenos fronterizos en los que los pedagogos encontraban lugares comunes y a los cuales se recurría sin acabar de delimitarse la autoría original de manera total. Los trasvases son evidentes desde la filosofía de unos métodos y otros (no olvidemos que, por ejemplo, el

8 GRAETZER, YEPES (1961)

9 Pensemos por ejemplo en el peso de Orff en la producción pedagógica del propio Jos Wuytack, quien se encargó al igual que Graetzer de su propia adaptación.

10 YEPES (1968)

11 SZÖNYI (1976). factor rítmico de Orff tiene una fuerte inspiración dalcroziana) y ello suponía alguna disfunción para el lector de lengua castellana, quien hubo de esperar a que esos parámetros quedaran reafirmados e identificados con autores concretos a través de estas monografías. Es el caso de los gestos fononímicos que hoy se entienden como parte integral del método Kodály y que, de manera explícita, Schoch atribuía a Josef Wenz:

En 1950, Josef Wenz, en su publicación Musikerziehung durch Handzeichen, Neuformung eines alten Weges ("Educación musical por signos manuales, renovación de un estudio antiguo") [...] basándose en reflexiones científicas y experiencias prácticas, propuso una reforma de los signos manuales para poder emplearlos también en la música moderna y alteraciones cromáticas. Como sus complementos han sido aceptados por renombrados pedagogos musicales, los publicamos aquí... ${ }^{12}$

No se trata, en este caso, de otro modelo fononímico, con diferentes gestos a los teorizados por Kodály (como encontramos, por ejemplo, en Justine Ward) sino, más bien, una identificación tal que sería difícil no afirmar cierto contacto en la gestación metodológica. Otro ejemplo válido nos lo aporta Kurt Pahlen, curtido en las escuelas germanas, quien ilustra procesos como la solmisación de Kodály sin llegar a citarlo de manera explícita ${ }^{13}$. Obviamente, como venimos señalando, hemos de pensar que estamos ante coetáneos que compartían planteamientos metodológicos lindantes los cuales, en determinados casos, podrían llegar a converger en lo que finalmente ha quedado en una aportación individual -lo cual esconde muchos matices- de un solo autor.

Por otro lado, es necesario resaltar que algunas de las elaboraciones pedagógicas más meritorias del presente se deben a personalidades que conocían a la perfección estas primeras aportaciones, ya que en su día colaboraron en la difusión de las adaptaciones a las que nos estamos refiriendo. La reiteración de las ideas de estos autores contribuyó de manera decisiva a la fijación estandarizada de las metodologías primeras ${ }^{14}$. Como señalamos, se trata de referentes en la pedagogía musical que, con el paso del tiempo y a raíz de su contacto directo con estas propuestas, generarían modelos propios aprovechando el conocimiento asimilado. Este es el

12 SCHOCH (1964): 19.

13 PAHLEN (1961): 27.

14 En nuestro contexto inmediato se conjugaron con otras aportaciones de corte más personal y clásico. $C f r$. TORRIJOS HORTELANO (1958); en las ediciones fuera de España pero en castellano se va de textos más generales a otros con prácticas muy específicas: $C f r$. GARCÍA ACEVEDO (1964). BAREILLES (1974). 
caso de Violeta Hemsy de Gainza ${ }^{15}$ en Argentina, quien por ejemplo encauzó los procesos de palabras rítmicas de Orff junto con la disposición de las alturas de sonidos con que Kodály comenzaba determinados saltos interválicos en canciones simples. La autora también se volcó en la traducción de derivaciones de la obra de Jaques-Dalcroze a partir de los planteamientos adaptados de Frances Webber Aronoff ${ }^{16}$. Al respecto de Jaques-Dalcroze, hay que señalar que sus ideas, con un fuerte impulso en la actualidad en determinados focos de la geografía española ${ }^{17}$, aun estando entre las primeras en florecer ${ }^{18}$ y mostrar ciertos paralelismos con otras obras dentro de estas épocas ${ }^{19}$, han encontrado un progreso significativo en forma de monografías en las últimas décadas ${ }^{20}$. En este sentido, el crecimiento de los centros especializados en su planteamiento rítmico ha hecho aumentar el conocimiento sobre su obra de forma considerable, en la teoría por medio de publicaciones y en la práctica a través de cursos de formación.

Por otro lado, la propia Hemsy de Gainza se entregó también a la difusión del método de Edgar Willems, de quien fue alumna, haciendo de puente entre la versión original y la castellanizada ${ }^{21}$. Es remarcable que, a pesar de que su obra, otra de las grandes aportaciones en torno a la fundamentación psicológica de la educación musical ${ }^{22}$, ya fuera accesible en la década de los sesenta, en realidad se reedita de manera más sistemática -incluso frenética- en castellano desde su muerte en 1978 (aunque también en otros idiomas, como los famosísimos cuadernillos en francés de las Éditions Pro Musica, puestos en circulación periódicamente ${ }^{23}$ ). Además de la de Willems, la producción de Maurice Martenot fue otra cuya traslación emergió a mediados del siglo pasado en

15 Sería difícil seleccionar alguna obra de la ingente producción de la autora argentina. En todo caso, para los propósitos de este texto rescatamos la filosofía de la propuesta de uno de sus textos más significativos. Cfr. HEMSY DE GAINZA (1970).

16 WEBBER ARONOFF (1974).

17 A partir, sobre todo, de los trabajos de Llongueres, preocupado por la explicación del método y la puesta en práctica del mismo a través de ejercicios y la continuidad metodológica de Benedito, junto con el impulso posterior sostenido hasta nuestros días del propio Instituto Llongueres. Todo ello es mostrado con detalle por Olga María Toro. $C f r$. LLONGUERES (1942); BENEDITO (1951); TORO EGEA (2010).

18 La compilación propia más referenciada suele ser la de la década de los veinte. C fr. JAQUES-DALCROZE (1920)

19 Cfr. COMPAGNON, THOMET (1966).

20 BACHMANN (1998); VANDERSPAR (1990).

21 Entre otras aportaciones en sus compilaciones de estudios es común encontrar elementos explicativos sobre las pautas seguidas por el pedagogo belga. $C f r$. HEMSY DE GAINZA (1977).

22 WILLEMS (1962); (1964a); (1964b); (1981).

23 Donde prologaron seguidores y pedagogos más actuales como Jacques Chapuis. forma de pequeños manuales ${ }^{24}$ (en algún caso bajo la traducción y adaptación de Adela Olivieri) y que, con alguna reedición intermedia, se difundió varias décadas después con las garantías de formatos más asentados ${ }^{25}$. Otros planteamientos que también han conservado cierta actualidad y a los que los docentes recurren con cierta asiduidad son los de Murray Schafer, cuyas ediciones clásicas siguen siendo bien consideradas $^{26}$. Algo menos de arraigo ha tenido el método de Justine Bayard Ward, el cual produjo mucha literatura desde sus trabajos vinculados al gregoriano de la década de 1920. Los intentos por establecerse como un referente de mayor calado vienen determinados por las publicaciones de $1964^{27}$. En todo caso, si en su origen la afinación en el canto podría engarzar con ciertos criterios desarrollados por otros autores, su potencial actual, que sigue latente, no ha enraizado de manera sólida en las diferentes etapas de la enseñanza obligatoria.

\section{DE LAS TRADUCCIONES, ADAPTACIONES Y CURSOS DE ESPECIALIZACIÓN}

Por lo general, desde mediados del siglo XX, un alto porcentaje de la bibliografía escrita para el público hispanohablante que versara sobre pedagogía musical habría de ser una traducción o adaptación, en la cual se referenciara alguno de los autores que luego formarían el núcleo duro de pedagogos con los que se interpretaría nuestra realidad metodológica años después, esto es, fundamentalmente aunque no exclusivamente Carl Orff, Zóltan Kodály, Émile JaquesDalcroze y Edgar Willems, entre otros.

En todo caso, aunque con el paso del tiempo se pudieran contar bastantes obras de referencia para la pedagogía musical en castellano a partir de traducciones o adaptaciones de manuales foráneos, en un primer momento no eran tantos los volúmenes editados. En este sentido, en los albores de la recepción, existió cierta preocupación por la ausencia de traducciones de textos que ilustrasen las nuevas ideas pedagógico-musicales. Este factor es notorio y no pasó inadvertido, particularmente, en los países sudamericanos donde se publicaban la mayoría de las obras que acabaron siendo referencias para España. Así lo plasmaba María Elena González al constatar que

en Europa se practican métodos para la enseñanza de la música que evidencian una gran preocupación por parte

\footnotetext{
24 MARTENOT (1957a); (1957b).

25 MARTENOT (1993).

26 MURRAY SCHAFER (1965); (1967); (1975).

27 WARD (1964a); (1964b).
} 
de los maestros para lograr una perfecta coordinación en todas las actividades que el niño desarrolla con fines educacionales. La enseñanza de la música no se considera una actividad aislada y específica de una sola clase; no tiene como finalidad formar músicos instrumentistas, sino contribuir a la formación integral del educando [...]

Desgraciadamente, en nuestro país [Argentina] estos métodos sólo se conocen en forma incompleta, fragmentaria, ya que la mayoría de ellos no están traducidos al castellano, ni aun al francés o al inglés, idiomas más accesibles para nosotros. Por otra parte, el dominio de estos métodos lo ejerce un número muy reducido de pedagogos y a pesar de toda su buena voluntad para la mayor difusión de los mismos, sólo es posible tener una idea aproximada de los procedimientos que se emplean en cada método a través de cursillos breves, tal vez demasiado breves, dados en algunas ciudades del país ${ }^{28}$.

La cita anterior, sumamente reveladora, se remata constatando que "en Europa" (circunscribiendo esta zona geográfica especialmente a Alemania, algunos países del Este y Francia) están en boga las teorías de Jaques-Dalcroze, Ward, Martenot y, el modelo más completo para la autora, $\mathrm{Orff}^{29}$. De sus postulados extraemos, por su potencial para nuestra argumentación, al menos tres ideas fundamentales: en primer lugar el cambio propiciado en Centroeuropa tiende a propagarse por la necesidad de poner en valor las ideas de globalidad, generalización de la educación, vivencia, ludicidad o creatividad que fluían desde estos modelos metodológicos; asimismo, se constata la dificultad para acceder a este tipo de conocimientos en formato bibliográfico, dada la traba idiomática que suponía para los lectores de habla hispana el alemán y, en su caso, otras lenguas como pudiera ser el húngaro; finalmente, la principal correa de transmisión no deja de ser el formato de cursos de formación, encuentros de pedagogos por varios países del continente y seminarios promovidos en diversos centros de enseñanza superior ${ }^{30}$.

Así, la primera idea que empezará a calar fue que el amparo perfecto para los fundamentos sociológicos, pedagógicos, psicológicos y epistemológicos de la nueva educación musical (la que venía, por ejemplo, de la apertura pedagógica propuesta antes, entre otros, por Montessori) iba a ser

28 GONZÁLEZ (1963): 1.

29 Otros autores de idéntico contexto geográfico, tomando fuentes similares, llegan a conclusiones parecidas. Cfr. SCIARRILLO GIANNEO, VIVERN DE SCIARRILLO (1979): 84-89.

30 En ese ámbito, por ejemplo, destacamos la figura de Ana Lucía Frega, al margen de la gran cantidad de producción (mucha de ella en formato de artículos) que ha ido produciendo a lo largo del tiempo. el que propiciara la evolución de la misma. En palabras de Bareilles y Zen:

Aunque estas recomendaciones parecieran banales, tienen su importancia en cuanto preservan al niño de la mala educación musical, que es la que se ajusta a los peores métodos de enseñanza basados en el dogmatismo, el empleo exclusivo de la memoria y la elección arbitraria de temas ${ }^{31}$.

Este novedoso predicamento pudo alcanzarse en España gracias a la movilidad de determinados músicos y pedagogos por el continente ${ }^{32}$, y llegó a otros países latinos, igualmente, por figuras que habían sido formadas en Centroeuropa pero que, por uno u otro motivo, culminaron su carrera y vida en otras latitudes. Especialmente reseñable es el caso del previamente citado Kurt Pahlen quien, tras huir de la persecución nazi en los prolegómenos de la Segunda Guerra Mundial, encontró refugio y espacio creativo en Argentina. Pahlen elaboró varios textos que sirvieron para anclar la filosofía de las pedagogías musicales en el público hispanohablante, así como escritos venidos de modelos ensayísticos $^{33}$. Afirmaciones que prologaban sus obras como "la finalidad de la educación musical no puede ser, en ningún momento, la de promover músicos", "la educación musical es para todos, absolutamente todos, sin excepción alguna", "la educación musical comienza en la cuna", "la existencia de tanta gente 'no musical' o 'amusical' [...] prueba únicamente la ineficacia de la enseñanza musical" o "la educación musical ha de dotar al niño de sensibilidad para captar las manifestaciones del mundo sonoro" 34 podrían haber sido suscritas por cualesquiera de los autores que venimos mencionando.

Pero, sin duda, la divulgación directa fue el condimento perfecto para hacer de este goteo de publicaciones un robusto conjunto procedimental de éxito. Aún hoy, por ejemplo, es común que se nos remita a las conferencias de Egon Kraus ${ }^{35}$ y Wilhem Twittenhoff en el marco de la mítica reunión científica que la Unesco auspició en Bruselas (1953) porque, en realidad, esa era la principal vía de adquisición de estas modernas aplicaciones pedagógico-musicales. Junto con el simposio anterior, el Congreso sobre Pedagogía Musi-

31 BAREILLES, ZEN (1964): prólogo, s. p.

32 Un caso como el descrito es Manuel Angulo, dedicado a varias facetas musicales durante su vida, entre ellas la pedagógica. Cfr. ANGULO (1968).

33 PAHLEN (1965).

34 PAHLEN (1961): 7-8.

35 Quien posteriormente trataría de mapear diversos modelos de enseñanza musical por el mundo. Cfr. KRAUS (1964). 
cal de Copenhague (1958), también bajo la organización de la Unesco, sirve para asestar los golpes de timón definitivos que canalicen las ideas que habrían de triunfar.

Por otro lado, en lo que atañe a la transmisión de los ideales de las enseñanzas de estos autores se ha hecho referencia expresa a la difusión a través de cursos formativos a los que solían acceder profesionales de alto nivel, no tanto docentes a pie de aula (de hecho la ausencia de la educación musical reglada en estos periodos tal como la conocemos hoy imposibilitaba tal circunstancia). Así por ejemplo, la propia Montserrat Sanuy elabora el material basado en Orff para el público español, el cual ha sido objeto de comentario, tras su paso por el Instituto Orff de Salzburgo y el contacto con González Sarmiento, quien dominaba más el idioma alemán ${ }^{36}$. Por tanto, sin la existencia de este sistema de talleres al que fueron confluyendo especialistas de diversos países no hablaríamos del auge que le siguió. A partir de este momento ya no sólo los propios autores vivos sino que, especialmente, sus seguidores, se dedicaron a urdir una red de transmisión muy tupida, que solía partir de núcleo de generación y se extendía rápidamente por todo el mundo.

El funcionamiento de estos cursos, como semillas que habrían de germinar en diferentes rincones que acogían estas ideas, queda plasmado en la siguiente cita en la que Madeleine Gagnard se aproxima a la transmisión del método Orff en Francia:

El método de Karl Orff fue introducido en Francia y adaptado a la lengua francesa por músicos jóvenes, que en 1965 siguieron un curso de dos meses en el Instituto Orff de Salzburgo, donde presenciaron el trabajo realizado por los niños con el equipo de profesores que perfeccionaron este método.

Desde 1965 los cursos se multiplicaron en París y en las provincias (hubo once); cada una de las personas que siguió el curso debía pasar todos los días por diferentes talleres: de pedagogía, de flauta dulce, de percusión (basada en la flexibilidad y la elasticidad del cuerpo), de expresión corporal. Así, se formaron cierta cantidad de profesores y este método, que hace maravillas, sobre todo con los niños pequeños, se difundió en Francia, en algunos conservatorios y en muchas escuelas primarias ${ }^{37}$.

De manera flexible, puede entenderse pues que la década de los sesenta supuso la puesta en funcionamiento de estos

36 Algunas de las motivaciones y hechos que llevaron a esa publicación pueden encontrarse en una entrevista a Montserrat Sanuy publicada en la revista "Música y Educación". Cfr. LAGO, PIÑERO, PLIEGO DE ANDRÉS (Madrid, 2007): 7-12.

37 GAGNARD (1973): 118-119. seminarios e institutos por Europa y que, la de los setenta, implementó el impulso para los simposia que han llegado hasta nuestros días. Aunque en España se atestiguan cursos más o menos focalizados acerca de estos autores, lo cierto es que los existentes son considerados esenciales ${ }^{38}$. La importancia de estos simposia en la generación de un conglomerado de actuaciones pedagógicas es más que evidente $\mathrm{y}$, en lo referente a casos concretos como el del "Método Kodály", puede hallarse un momento de ebullición que se toma como inflexión al alza. En este sentido se trataría de la celebración de un congreso específico por parte de la Sociedad Internacional de Educación Musical en la ciudad de Budapest en 1964 ${ }^{39}$. Si el crecimiento se entiende como sostenido desde las primeras aportaciones del pedagogo húngaro, desde este evento dicho crecimiento ha de considerarse exponencial.

Entre los comentarios documentados por ponentes en los medios de comunicación, destacamos el de László Eös$\mathrm{ze}^{40}$, quien en 1978 recaló en Madrid para impartir cursos en torno a la figura de Kodály (muerto diez años antes):

...existe ya una Sociedad Internacional Kodály, con sede en Budapest. Tenemos más de trescientos miembros por todo el mundo. Yo vengo de Canadá, donde nos hemos reunido en un congreso, ya el tercero, y el próximo se realizará en Sidney porque la presidenta de la Sociedad Internacional reside en esta ciudad australiana. Después de Sidney iremos a Tokio ${ }^{41}$.

Queda patente pues la importancia decisiva de estos cursos de formación en el proceso que estamos describiendo. Desgraciadamente, a pesar de que España acogiera este tipo de eventos (no deja de llamar la atención de nuevo la tardía llegada de los mismos), la ausencia de una infraestructura sólida -al menos en ese momento- que mantuviese viva a través de la práctica los planteamientos que llegaban, hizo que su desarrollo no fuese el deseado, como sí lo fue en otras latitudes, donde podemos hablar de auténtica ebullición.

\section{DE LOS TRÁNSITOS HACIA UNA PEDAGOGÍA MUSICAL AUTÓCTONA}

Poco a poco, el filtrado de estas ideas genera un poso que impregna los procesos propios de la pedagogía musi-

38 AYMERICH, AYMERICH, BUSQUÉ (1971): 12-13.

39 Dicho punto de inflexión es marcado por determinados autores, enfatizando la difusión posterior, como es el caso del prólogo de Manuel Angulo. Cfr. SÁNDOR (1981): 8. (1962).

40 Destacamos su mayor aportación al respecto: $C f r$. EÖSZE

41 RUIZ TARAZONA (Madrid, 1978). 
cal y, como es de esperar, los educadores van adaptando sus criterios a la filosofía general que ha ido penetrando capilarmente ${ }^{42}$. Sea como fuere, aceptada la adquisición parcial de conocimientos a partir de una bibliografía no tan amplia como se querría y de unos cursos constantes pero no siempre accesibles, a finales de la década de 1970 se puede afirmar que se ha establecido, al menos en los altos estratos docentes, una conexión mínimamente fructífera con los métodos de la primera parte de siglo. Sin embargo, las posturas en torno a su aceptación no siempre son uniformes. Por lo general se observa la necesidad de adaptación a nuestro contexto, una tendencia lógica que imbuye con más fuerza a algunos autores, hasta el punto de parecer desechar conceptos integrados durante estos años:

Nuestra experiencia de varios años nos ha llevado a la conclusión de que la escuela no admite un método definido y concreto de música ya elaborado, porque no intenta formar virtuosos de la música, sino proporcionar mediante ella al niño los aspectos que le falten para lograr su equilibrio interno, y con ello una apertura hacia el entorno, que sólo se consigue desarrollando la sensibilidad. Cada individuo tiene unas necesidades distintas, no sirve para todas las puertas la misma llave ${ }^{43}$.

En todo caso, si desgranamos las características de la propuesta del autor anterior, la filosofía que muestra a lo largo de su trabajo deja entrever elementos tan reconocibles que no puede obviarse el contacto metodológico con las pedagogías precedentes y su puesta en práctica de un modo diferente si se quiere. La principal dificultad que puede observarse es, por tanto, la ausencia -todavía- de una infraestructura sólida en lo referente a materiales y una red ensamblada de profesionales bien formados en el plano musical para el trabajo en los centros educativos:

Sería, realmente, perfecto que la escuela pudiese enseñar a los niños a tocar los formidables instrumentos de percusión que hoy existen, o el delicioso instrumento de la flauta, tan simple y tan perfecto. Pero comprendemos que no es cosa posible [...]. Como es también difícil encontrar profesorado especializado capaz de manejar bien estos instrumentos ${ }^{44}$.

42 Ejemplo de ello podría ser el conjunto de ritmos (muy similares a la propuesta inicial de Orff) y cantos y recitados de algunas publicaciones en España de Peris o las aplicaciones metodológicas de Veltri. $C f r$. PERIS LACASA (1965). VELTRI (1969).

43 MORENO (1979): 47.

44 AYMERICH, AYMERICH, BUSQUÉ (1971): 11.
Las publicaciones que tienen formato de manual para la docencia en torno a la década de los setenta suelen caracterizarse por plantear ejemplos inequívocamente vinculados a algún método que posea el carácter indiscutible, ganado por la propia fama que le antecede. En este sentido, es habitual que la firma a este tipo de volúmenes la estampe alguna personalidad que ocupe un puesto relevante en la docencia universitaria o en la propia administración educativa ${ }^{45}$. Es el caso, por ejemplo, de Matilde Murcia ${ }^{46}$, quien en sus dos volúmenes para la formación musical se hace eco de los parabienes de las instrumentaciones Orff. Otra catedrática como ella, María Cateura ${ }^{47}$, aparecerá entre las referencias más notorias en el contexto español en lo que atañe a manuales pedagógico-musicales que acomodan los materiales preexistentes y comienzan a proyectarlos al futuro. Todavía lejos del cambio de modelo educativo hacia una enseñanza obligatoria con una educación musical consolidada, la autora realiza una traslación por niveles de las actividades musicales que pueden tener cabida en nuestro contexto más inmediato, basándose en la propia experiencia y observación directa:

Lo publicado en España hasta el presente [1976] sobre la didáctica de la Música, con todo su indudable mérito que somos los primeros en reconocer, queda aún bastante lejos de lo que se publica en otros países europeos.

En las publicaciones sobre Música para la infancia se viene observando una cierta disparidad de enfoques [...]. Hemos podido apreciar que en los colegios, aún disponiendo de tales libros, muchos maestros no se deciden a impartir la enseñanza de la Música a sus alumnos si no dominan el solfeo, y, los que saben algo, realizan alguna tarea esporádica de enseñanza [...].

Esta labor en la E.G.B., bien realizada, creo que es uno de los mejores caminos de acercamiento al nivel musical de los centros docentes europeos ${ }^{48}$.

Su texto, en el ámbito rítmico, anda repleto de ejercicios imitativos, palabras y sílabas rítmicas; en el melódico, de modelos fononímicos y representacionales; en el del movimiento, de desplazamientos, actividades de lateralidad y expresión corporal; en el instrumental, toma en consideración

45 En ese contexto se enmarcan las aportaciones de Rosa María Kucharski, quien tras una sólida formación pianística se vinculó a la pedagogía musical involucrándose en la gestación y presidencia de la Sociedad de Educación Musical y colaborando con el Ministerio a través de su Servicio de Orientación Didáctica. Cfr. KUCHARSKI (1974); (1980).

46 MURCIA (1976); (1979).

47 CATEURA MATEU (1976a); (1976b).

48 CATEURA MATEU (1976a): prólogo, s. p. 
la flauta dulce además de la pequeña percusión de manera prototípica $^{49} \ldots$ Se trata, en suma, de una adaptación a la realidad más cercana a través de procedimientos válidos y consistentes para su aplicación en la España que transitaba por la Ley-Plan 1967. Los pedagogos presentes en el volumen citado, sin ser mencionados, son claramente identificables ya $\mathrm{y}$, por tanto, se puede entender que se empieza a avanzar en la plasmación directa de lo que con posterioridad, en torno a quince años después, se convertirá en dotación material y sistematicidad en su uso. No es baladí, y por tanto no debe ser obviada, la última frase de la autora: la aspiración a mejorar la enseñanza de la música a partir de procedimientos concretos puesto que ello, desde esta perspectiva, acabaría por configurar cambios estructurales hacia una educación musical más anclada en la enseñanza general, de acuerdo con los modelos de los países europeos de referencia.

Complementando la obra anterior con musicalizaciones más sistemáticas y ahondando en los ejemplos para el aprendizaje de la música vocal, la publicación de 1979 por parte de Nicolás Oriol y José María Parra se preocupó, igualmente, de recoger la casuística de actividades más aprovechables desde el punto de vista metodológico y acercarlas hacia las leyes educativas vigentes en España. De este modo, los anexos referidos a objetivos, contenidos, actividades, técnicas, material y evaluación, aunque obviamente desfasados a día de hoy, nos muestran la intención clara de encarrilar la metodología hacia una realidad concreta. La bibliografía manejada para llevar a cabo la obra constata hasta qué punto son tenidas en cuenta las traducciones de las dos décadas anteriores (sesenta y setenta) así como obras de menor entidad también en castellano. A partir de este texto, de gran difusión en su día, se empezará a atisbar un periodo en el que nos hallemos a las puertas del punto de inflexión, el que marcará la graduación de un buen número de docentes especializados en música, en el momento en que la legislación española incluirá este perfil dentro de su organigrama educativo.

En realidad, a pesar de que la pedagogía musical se incluyera en los estudios de los conservatorios desde el antiguo Plan 66 y que el cambio legislativo intentase paliar las dificultades venidas del ámbito pedagógico ${ }^{50}$, no fue en esa esfera donde los métodos transmitidos encontraron aco-

49 En las obras posteriores dedicadas al público infantil, la autora reproducirá dichos criterios, especialmente en el ámbito vocal y rítmico simple. Cfr. CATEURA MATEU (1982).

50 Los lamentos al respecto pivotaban en torno a la carencia de herramientas conceptuales en el plano pedagógico de los profesionales que salían titulados de los conservatorios, lo cual se unía a otro tipo de carencias materiales que se pusieron de manifiesto en este tránsito. $C f r$. ESTÉVEZ VILA, 49 (Madrid, 2002): 48. modo. Tampoco suele otorgarse una importancia capital al hecho de que las Orientaciones Pedagógicas, aprobadas en 1970, contemplasen dentro de un amplio haz de materias el desarrollo musical, si bien llama la atención que ya entonces se refería la necesidad de, de uno u otro modo, aproximarse a autores como Ward, Kodály u Orff ${ }^{51}$. Cierto es que el carácter obligatorio de la enseñanza de la música comienza a plasmarse por aquel entonces ${ }^{52}$, pero no es menos cierto que, en la práctica, la ausencia de voluntad y de docentes preparados para la impartición de la materia cercenaba las potencialidades de la pedagogía musical en este contexto. En los siguientes amargos términos se quejaba Montserrat Casas, la prologuista del libro de recursos musicales de $\mathbf{M}^{\mathrm{a}}$ Teresa Giménez Morell, durante el periodo al que nos estamos refiriendo:

Estoy convencida de que somos muchos los maestros que creemos en el gran valor educativo de la música, pero nuestros conocimientos no nos permiten hacerla llegar a la escuela. Quizá nos atrevamos a cantar alguna canción si antes la hemos aprendido muy bien, pero no somos capaces de preparar una audición de modo que resulte útil para la formación de los niños ${ }^{53}$.

En el mismo sentido, Montserrat y Conchita Sanuy, tras reconocer que toda la aportación que hacen es heredera directa de las enseñanzas del Orff-Schulwerk, constatan las trabas para ponerla en funcionamiento por cuestiones meramente operativas:

Al entrar el maestro en el ejercicio de su profesión, no cuenta con base para poner en práctica las actividades musicales, cosa que no le ocurre con otras materias. Si a esto añadimos que muchos maestros creen que no tienen condiciones específicas para la música, comprenderemos la razón por la que en muchas escuelas no se desarrollan estas actividades ${ }^{54}$.

Aún más expedito es el comentario de Jesús Iglesias, quien desde su condición de maestro explicaba la delicada situación previa a la década de los noventa:

A mediados de la década de los 80 el MEC [...] convocó algunos Cursos de Monitores de Educación Musical,

51 Cfr. VICENTE GUILLÉN, VILLENA RAMÍREZ, 15 (Murcia, 1997): 86.

52 ORIOL (1979): 14.

53 Extracto del prólogo de Montserrat Casas y Vilalta. Cfr. GIMÉNEZ MORELL (1984): 14.

54 SANUY, SANUY (1982): 17. 
posteriormente efectuó una convocatoria de la Formación de Formadores en E. Musical, y por último, diseñó una serie de cursos de Especialización Musical en convenio con las Universidades [...]. Hasta esa fecha, ni el MEC ni las editoriales (porque no era negocio), editaban materiales apropiados para la asignatura, por lo que se entorpecía la labor de los maestros voluntarios que intentaban impartirla ${ }^{55}$

Con todo, es comúnmente aceptado que, para el caso de la música, el espaldarazo que supuso la Ley Orgánica General del Sistema Educativo (1990) no ha tenido parangón en la enseñanza obligatoria ${ }^{56}$. Sin duda, aunque a dicha Ley se le pueda achacar otra serie de carencias en diversos ámbitos, lo cierto es que facilitó que se articulase un espacio mínimamente serio para la música en la educación general. Como apuntamos antes, ese fue el punto de partida, igualmente, de la formación de maestros en este ámbito, los cuales aprendieron de docentes que habían interiorizado, si bien de manera dispar, todo el bagaje que hemos descrito con anterioridad. Así, los escritos relativos a Orff, Kodály o Jaques-Dalcroze tomarían un nuevo vigor al transmitirse a la incipiente hornada de profesores. Es importante tener en cuenta que, en ese momento, se empieza un proceso de configuración de las aulas de música en muchos lugares de la geografía española. Tal proceso fue, por otro lado, muy desigual, ya que la celeridad con que en determinados centros se dotaba de materiales era sumamente inferior a la de otros.

Tras este serpenteante recorrido, al llegar la década final del siglo XX eran varios los escritos que ya habían abonado el terrero del desarrollo metodológico señalado. Así, por ejemplo, el hecho de que Real Musical editara por aquel entonces compilaciones como la de Sándor ${ }^{57}$, que continuaba haciendo accesibles los planteamientos musicales venidos de Hungría con Kodály, parecía facilitar la labor de enseñanza a los futuros docentes. Sin embargo, se reproduce de nuevo la problemática, tal vez más acuciada, de la implantación de los modelos foráneos a un sistema educativo que estrenaba ahora, muchos años después, sus propios condicionantes.

Del mismo modo, desde Hispanoamérica, especialmente en zonas donde tendencias como el indigenismo po-

55 Intervención en la mesa redonda del evento señalado. $C f r$. SUSTAETA, ORIOL (1998): 62.

56 El Artículo 16 de la LOGSE contempla la figura del profesor especialista de educación musical, toda una novedad respecto a leyes anteriores. La orden del 19 de abril de 1990 genera el nicho laboral pertinente y establece los modos de habilitación para el profesorado que hubiera de cubrir esas primeras plazas, a partir de lo cual se perfilan las oposiciones para maestros de música.

57 SÁNDOR (1981). dían tener cierto empuje, se vuelve a cuestionar el uso metodológico alejado de la realidad misma de la zona. Conocidos los autores que renovaron la pedagogía musical, la filosofía de algunos de los textos que adaptan sus criterios no dejan de criticar la manera de asumir irracionalmente cada uno de sus postulados sin un proceso de reflexión previa que lleve a modelar los elementos metodológicos más funcionales:

Una cosa es enriquecer nuestros conocimientos con lo logrado en otros países, y otra muy distinta es usar métodos y procedimientos de otros lados en los cuales, únicamente, ha mediado una adaptación directa y una traducción literal. Es incluso, frecuente, la incorporación en este tipo de trabajos, de rimas y música infantiles del país que adopta el método buscando una afinidad mayor entre el educando y las actividades del método importado. Este esfuerzo es inoperante. Se ha cambiado la vestidura pero no la médula ${ }^{58}$.

En este sentido, incluso las primeras obras que se hacían eco y promocionaban los métodos más difundidos poseían, por lo general, un sesgo que les hacía mostrar distancia (en algunos casos más que en otros) con aspectos sociales eminentemente diferenciales entre los países centroeuropeos y la audiencia potencial que leería la traducción de dichas obras. A pesar de los esfuerzos por aclimatar estas traslaciones, no siempre se podía buscar una correspondencia directa entre las ideas planteadas y la realidad musical del receptor del texto. Pensemos en la publicación en España de la obra de Margrit Küntzel-Hansen ${ }^{59}$, acerca de la enseñanza de la música a partir de actividades concretas para el desarrollo auditivo en los más pequeños, donde encontramos muy pocos anclajes directos para nuestro contexto (se muestra, por ejemplo, la manera de aproximar al niño a un instrumento a partir del uso del mismo por parte de los padres). Las diferencias con el territorio español eran evidentes. Sin embargo, aunque palpable, este aspecto nunca fue tratado con demasiada vehemencia desde España. Los propios Oriol y Parra realizan un breve recorrido por el tratamiento musical de los países del entorno europeo dejando constancia de la inevitable diversidad pero de forma bastante aséptica, sin criticar tal circunstancia ${ }^{60}$. Dichas aproximaciones laxas eran seguidas por otras monografías más amplias y concienzudas (trabajos doctorales) que tenían como piedra angular las prácticas externas e internas ${ }^{61}$.

58 TORT (1978): 8

59 KÜNTZEL-HANSEN (1981).

60 ORIOL, PARRA (1979): 13-14.

61 CATEURA MATEU (1992). 
En efecto, sucedió que, de manera más natural, en España se abrió un proceso de interpretación de procedimientos cotidianos en las aulas de música que hizo que determinados pedagogos comenzasen a ponderar las actividades que se adherían a la realidad inmediata ${ }^{62}$. Los propios defensores y difusores de estas referencias pedagógicas, empiezan a mostrar (si cabe con más intensidad) la necesidad de adaptar unas propuestas alejadas física y temporalmente de nuestros centros educativos. En ese momento paradigmático de eclosión es especialmente reseñable el alegato de Montserrat Sanuy quien, sin dejar de aprovechar para su propuesta lo mejor de los discursos anteriores, reflexiona sobre ellos del siguiente modo:

Existen muchas publicaciones sobre educación musical, aunque en su mayoría se trata de traducciones. Libros publicados originalmente en otros países en los que la música está presente y enraizada desde siempre en la escuela y, más aún, en la misma familia. Asimismo, los maestros que imparten esta materia, la disfrutaron también en su infancia. Todo esto hace suponer que existen unas condiciones (terreno abonado) que, a la hora de la verdad, no se corresponden hoy con las de nuestro país. Incluyen además, tradiciones, juegos, canciones, danzas e instrumentos ajenos a nuestra cultura ${ }^{63}$.

No es preciso recordar que comenzábamos esta aproximación señalando que Sanuy, junto con González Sarmiento, fue la encargada de aproximar en formato de pequeño manual las ideas de Orff, allá por 1969. Es sumamente relevante entender que, desde esta perspectiva, las referencias primeras siguen teniendo un peso capital en obras como la citada (el cual se atestigua, especialmente, en las musicalizaciones y el modo de ejemplificar las "palabras tipo para formar compases" ${ }^{64}$ ), pero en la configuración de estos textos comienza a forjarse claramente un buen paquete de prácticas asidas a una realidad educativo-musical contextualizada.

En ese momento, los nuevos recursos a nuestro alcance hacen que reconsideremos el papel de los métodos tradicionales y se busque su utilización como complemento de un plan general transversal. Conscientes de que se está ante el camino más fructífero, algunos de los seguidores de los autores abordados lo reflejaban sin paliativos:

62 Un caso particular es el de Luis Elizalde, pionero en la filosofía de acercarse vivamente al entorno más próximo en sus prácticas pedagógicas. Cfr. ELIZALDE (1979). Con la colaboración de Eva García-Bernalt.

63 SANUY (1994): 13.

64 SANUY (1994): 135.
El presente manual es un reflejo, expuesto en forma de notas, de cómo he venido utilizando dichas ideas hasta el momento. Los lectores pueden manejarlo con entera libertad y desarrollarlo para lograr una formulación propia $^{65}$.

Igualmente, las obras de tratamiento musical general no dejan de lado, durante los primeros años de aplicación de la LOGSE, que toda actividad propuesta bebe, en cierto sentido, de un fondo común, que aunque pueda estar ya muy alejado temporalmente sigue conservando evidentes vínculos con él:

Desde el punto de vista del tratamiento musical, las canciones que presentamos pretenden hacer hincapié en los elementos musicales básicos, como son la percepción del ritmo (pulso, compás) y de la melodía (frase y línea melódica), pero conducidos a través de una pedagogía activa, como bien sugieren el método Dalcroze o el método Orff, los cuales orientan la enseñanza de la música desde el movimiento ${ }^{66}$.

Tras hacer bastantes cursos y cursillos sobre pedagogías principales aplicadas a la música, me lancé al mundo de la enseñanza. He de confesar que, además de los métodos tradicionales que me enseñaron de pequeñita, que hoy no valdrían, pero que algo hicieron en su momento, estoy enteramente reconocida a muchos pedagogos. Sobre todo a K. Orff, Z. Kodály, J. Dalcroze, E. Willems y J. Withak [sic.]. Ellos me dieron el mejor punto de arranque que podría tener, y por ellos estoy a gusto en el aula con los pequeños $^{67}$.

En este momento, toda nueva aportación o idea con la que rematar un método, si se quiere fundamentar en sólidos criterios, busca su amparo en los modelos anteriores, entendiendo que aunque actualizable su filosofía tiene sentido y vitalidad ${ }^{68}$. Dicha ligazón continúa, durante esta implantación progresiva de la LOGSE, desde las aulas universitarias. Por citar algún ejemplo referente de la afirmación anterior, señalamos que las "Jornadas de Educación Musical: Música en la Educación Primaria" en las cuales parti-

65 VANDERSPAR (1990): 5.

66 CONDE CAVEDA, VICIANA GARÓFANO, CALVO NIÑO (1999): 12.

67 DE CEA (1994): 6.

68 Pensemos, por ejemplo, en los libros de texto con que María Pilar Escudero va elaborando sus propuestas o en la publicación del llamado "Método intuitivo de audición musical", el cual se enfoca teniendo en cuenta los avances de Jaques-Dalcroze, Orff o Kodály. Cfr. HERNÁNDEZ MORENO (1992): 13-16. 
ciparon algunos de los docentes universitarios señeros en este periodo de asentamiento legislativo, se continua incidiendo en los referentes metodológicos de la primera mitad de siglo XX. Así, Carmen Portela (Universidad de Santiago de Compostela) recordaba los intercambios de profesorado para el aprendizaje de las técnicas del Método Kodály o la impartición de dicha metodología en las asignaturas universitarias de Didáctica de la Educación Musical I y II ${ }^{69}$; Vicente Sanjosé (Universidad de Valencia), reconociendo graves dificultades en diversos órdenes, manifiesta su satisfacción por la dotación de las aulas con material Orff ${ }^{70}$; y Manuel Fernández (Universidad de Castilla - La Mancha), mostró experiencias en formato de curso con especial atención a los criterios desarrollados por Orff, Kodály, Willems, Martenot o Dalcroze ${ }^{71}$. En esencia, como se infiere de reuniones científicas como la anterior, las nuevas generaciones de maestros de música -las primeras en realidad para la escuela primaria- se instruirían en estos modelos pedagógicos, los cuales, tantos años después, poseían todavía un inmenso potencial que, en su proyección al presente, ha de remozarse constantemente de acuerdo con los cambios sociales y tecnológicos.

\section{CONCLUSIONES}

A pesar de la dificultad para hablar en términos generales de un contexto tan variopinto como el español en cuanto a aspectos educativos, de manera bastante uniforme se pueden encontrar correlaciones en la conformación de las aulas de música de nuestro país. Es difícil no hallar algún tipo de instrumentación Orff (al margen de que su uso sea más o menos frecuente), como tampoco lo es observar desarrollos metodológicos basados en su rítmica, la de Jaques-Dalcroze o los aspectos vinculados al canto propios de Kodály, por señalar los elementos más recurrentes. Del mismo modo, no es descabellado pensar que los propios libros de texto, que han sido alumbrados bajo el mismo influjo, hayan evolucionado desde patrones similares.

Sin ánimo de emitir juicios de valor en torno a la pertinencia metodológica en el plano musical de las aulas actuales, el contexto descrito nos llevó a plantearnos la necesidad de investigar en torno a los motivos que nos han conducido hasta este punto. Por un lado, se constata como elemento de vital importancia el contacto directo de pedagogos musicales españoles que viajaron en busca de

69 Intervención en la mesa redonda del evento señalado. $C f r$. SUSTAETA, ORIOL (1998): 14

70 SUSTAETA, ORIOL (1998): 25.

71 SUSTAETA, ORIOL (1998): 50. los ecos de unas metodologías que estaban cambiando los parámetros de enseñanza musical más allá de los Pirineos. Estas primeras influencias dieron como resultado traducciones y adaptaciones que fueron muy influyentes y que hoy en día se han convertido en clásicos por su valor referencial. Junto con estas publicaciones hay otro conjunto de textos en castellano que, igualmente, facilitan mucho la propagación metodológica: las ediciones desde Latinoamérica. Con especial énfasis en Argentina, a través de esos manuales se consigue que figuras que hoy día son señeras en la pedagogía musical desarrollen las ideas de estos pedagogos europeos, de forma que las marcas editoriales Eudeba, Ricordi o Kapelusz se conviertan en parte fundamental para el abordaje de los autores anteriores y otros igualmente reseñables, como es el caso de Willems.

De forma paralela a esas primeras monografías a uno y otro lado del Atlántico, se inicia la impartición de cursos, ya no sólo a cargo de los pedagogos más representativos sino por parte de sus seguidores más inmediatos, lo cual hace que se propaguen los métodos en cuestión de manera más vivaz. Otras personalidades de la pedagogía musical, por lo general desde el mundo universitario, toman contacto y reelaboran actividades a los que dan formato de manuales pedagógicos. La aceptación de la emergente filosofía dota de un renovado vigor a los materiales, que tratan de presentarse y adaptarse a partir de melodías autóctonas y, por consiguiente, cercanas al alumnado.

Sin embargo, el principal problema será que la legislación española, en el ámbito de la pedagogía musical, no avanza al ritmo que las tendencias didácticas que surgieran en Francia, Alemania o Hungría. Los materiales elaborados y alumbrados en forma de libros para la docencia no consiguen penetrar con todo su potencial en las aulas porque, si bien de manera sostenida desde la década de los setenta se tiende a ir habilitando espacios para la impartición de la música en las enseñanzas obligatorias, el análisis realizado y los testimonios rescatados nos hacen ver que, en realidad, hasta la habilitación de especialistas en la enseñanza de la música, allá por la década de los noventa, no se consiguió regularizar en cierto modo un proceso de enseñanza - aprendizaje musical mínimamente sostenido en el tiempo. Estos nuevos maestros, surgidos de la reforma acaecida con la LOGSE, se nutrirán de parámetros teorizados a lo largo de toda la centuria. Tan válidos como antiguos, es función de los docentes del futuro asegurar la actualidad de los mismos poniendo en valor sus virtudes desde su adaptación a la sociedad del presente. 


\section{BIBLIOGRAFÍA}

Angulo, Manuel, Música y didáctica. Madrid, Magisterio Español, 1968.

Aymerich, Carmen, Aymerich, María y Busqué, Montserrat, Expresión y arte en la escuela III. La expresión musical. La expresión como auxiliar didáctico. Barcelona, Teide, 1971.

Bachmann, Marie-Laure, La rítmica Jaques-Dalcroze. Una educación por la música y para la música. Madrid: Pirámide, 1998.

Bareilles, Oscar S., Educación musical. Buenos Aires, Kapelusz, 1974.

Bareilles, Oscar S., Zen, Norberto V., La música en el aula. Buenos Aires, Kapelusz, 1964.

Benedito, Rafael, Iniciación musical en la infancia. Madrid, Tipografía Artística, 1951.

Cateura Mateu, María, Por una educación musical en España: Estudio comparativo con otros países. Barcelona, PPU, 1992.

Cateura Mateu, María, Música. Nivel 1. Barcelona, Daimon, 1982.

Cateura Mateu, María, Formación musical en la educación básica. Niveles I-II-III. Barcelona, Clivis, 1976a.

Cateura Mateu, María, Formación musical en la educación básica. Niveles $I V-V$. Barcelona, Clivis, $1976 \mathrm{~b}$.

Compagnon, Germaine y Thomet, Maurice, Educación del sentido rítmico, Buenos Aires, Kapelusz, 1966.

Conde Caveda, José Luis, Viciana Garófano, Virginia y Calvo Niño, María Luisa, Nuevas canciones infantiles de siempre. Propuestas para la globalización de los contenidos expresivos en educación infantil y primaria. Archidona (Málaga), Aljibe, 1999.

De Cea, Lola, Guía didáctico-musical. Enseñanza primaria. Madrid, Mundimúsica, 1994.

Elizalde, Luis, Pedagogía del canto escolar. Madrid, Publicaciones Claretianas, 1979. Con la colaboración de Eva García-Bernalt.

Eösze, László, Zoltán Kodály. His life and work. Londres, Collet's Holdings LTD, 1962.

Estévez Vila, Jaime, "Del Plan 66 al Plan LOGSE: Aspectos positivos y problemáticos", Música y Educación, 49 (Madrid, 2002): 45-60.

Font Fuster, Rosa, Metodología del ritmo musical. Madrid, Ediciones Paulinas, 1980.

Gagnard, Madeleine, Iniciación musical en la enseñanza primaria y secundaria. Buenos Aires, Paidós, 1973.

García Acevedo, Mario, Didáctica musical. Ricordi, 1964.

Giménez Morell, María Teresa, La música en la escuela. Barcelona, Paidós, 1984.
González, María Elena, Didáctica de la música. Buenos Aires, Kapelusz, 1963.

Graetzer, Guillermo, Orff-Schulwerk (Obra didáctica de Carl Orff). Música para niños. Buenos Aires, Barry, 1961.

Graetzer, Guillermo y Yepes, Antonio, Introducción a la práctica del Orff-Schulwerk. Buenos Aires, Barry, 1961.

Hemsy de Gainza, Violeta, Fundamentos, materiales y técnicas de la educación musical. Ensayos y conferencias: 1967-1974. Buenos Aires, Ricordi, 1977.

Hemsy de Gainza, Violeta, La iniciación musical del niño. Buenos Aires, Ricordi, 1970.

Hernández Moreno, Antonio, Música para niños. Aplicación del 'Método intuitivo de audición musical' a la educación infantil y primaria. Madrid, Siglo XXI, 1992.

Jaques-Dalcroze, Émile, Le rythme, la musique et l'éducation. París, Librairie Fischbacher, 1920.

Kraus, Egon, El estado actual de la educación musical en el mundo. Buenos Aires, Eudeba, 1964.

Kucharski, Rosa María, La música, vehículo de aprendizaje cultural. Madrid, Ministerio de Cultura, 1980.

Kucharski, Rosa María: Música para las aulas. Madrid, Relieves Arsango, 1974.

Küntzel-Hansen, Margrit, Educación musical precoz y estimulación auditiva. Barcelona, Editorial Médica y Técnica, 1981.

Lago, Pilar, Piñero, Cecilia y Pliego de Andrés, Víctor, "Entrevista con Montserrat Sanuy", Música y Educación, 70 (Madrid, 2007): 7-12.

Llongueres, Joan, El ritmo en la educación y la formación general de la infancia. Barcelona, Labor, 1942.

Moreno, Miguel A., Música y expresión dinámica en E.G.B. Madrid, Narcea, 1979.

Murcia, Matilde, Formación musical. $2^{\circ}$ curso. Madrid, Real Musical, 1979.

Murcia, Matilde, Formación musical. 1er curso. Madrid, Real Musical, 1976.

Murray Schafer, Raymond, El rinoceronte en el aula. Buenos Aires, Ricordi, 1975.

Murray Schafer, Raymond, Limpieza de oídos. Buenos Aires, Ricordi, 1967.

Murray Schafer, Raymond, El compositor en el aula. Buenos Aires, Ricordi, 1965.

Nettl, Bruno, "Reflexiones sobre el siglo XX: el estudio de 'Los Otros' y de 'nosotros' como etnomusicólogos", Revista Transcultural de Música, 7 (Barcelona, 2003)

Orff, Carl y Keetman, Gunild, Die Weihnachtsgeschichte. Orff-Schulwerk Jugendmusik. Mainz, Schott, 1952.

Oriol, Nicolás: "La música en las Enseñanzas de Régimen General en España y su evolución en el siglo XX y comienzos del XXI", LEEME 16 (Valencia, 2005) 
Oriol, Nicolás, Parra, José María, La expresión musical en la educación básica. Madrid, Alpuerto, 1979.

Pahlen, Kurt, El niño y la música. Buenos Aires, El Ateneo, 1965.

Pahlen, Kurt, La música en la educación moderna. Buenos Aires, Ricordi, 1961

Peris Lacasa, José, Música para niños. Madrid, Doncel, 1965.

Ruiz Tarazona, Andrés, "Una forma de aprender música: el sistema Kodály”, El país, (Madrid: 7/6/78)

Sándor, Frigyes (ed.), Educación Musical en Hungría. Madrid, Real Musical, 1981.

Sanuy, Montserrat y González Sarmiento, Luciano, OrffSchulwerk Música para niños. Introducción. Madrid, Unión Musical Española, 1969a.

Sanuy, Montserrat y González Sarmiento, Luciano, OrffSchulwerk. Música para niños I. Madrid, Unión Musical Española, 1969b.

Sanuy, Montserrat, Aula sonora (Hacia una educación en primaria). Madrid, Morata, 1994.

Sanuy, Montserrat, Sanuy, Conchita, Música, maestro. Bases para una educación musical. 2 - 7 años. Madrid, Cincel, 1982.

Schoch, Rudolf, Educación musical en la escuela. Buenos Aires, Kapelusz, 1964.

Sciarrillo Gianneo, Rogelio C. y Vivern de Sciarrillo, Carmen F. M., La educación musical en la escuela primaria. Buenos Aires, Víctor Leru, 1979.

Sustaeta, Ignacio y Oriol, Nicolás (coord.), Actas de las Jornadas de Educación Musical. La música en la educación primaria. Madrid, Universidad Complutense, 1998.

Szönyi, Erzsébet, La educación musical en Hungría a través del método Kodály. Budapest, Corvina, 1976.
Torrijos Hortelano, Esteban, La música y el canto en la Escuela Primaria. Barcelona, Salvatella, 1958.

Toro Egea, Olga María, Documentación de materiales para la enseñanza de la música en España (1823-1932): catalogación, análisis y estudio. Córdoba: Servicio de Publicaciones de la Universidad de Córdoba, 2010.

Tort, César, Educación musical en el jardín de niños. México, Universidad Nacional Autónoma de México, 1978.

Vanderspar, Elizabeth, Manual Jaques-Dalcroze. Barcelona: Ed. Pilar Llongueres, 1990.

Veltri, Alicia Leonor, Apuntes de didáctica de la música. Barcelona, Daiam, 1969.

Vicente Guillén, Antonio y Villena Ramírez, María Isabel, "La educación musical, disciplina curricular en la formación del docente primario. Aportaciones legislativas: Siglos XIX-XX”, Anales de Pedagogía 15 (Murcia, 1997): 75-88.

Ward, Justine, Método Ward. Pedagogía musical escolar. París, Desclée, 1964a.

Ward, Justine, Método Ward. Primer año, libro del maestro. Bilbao, Desclée, 1964b.

Webber Aronoff, Frances, La música y el niño pequeño. Buenos Aires, Ricordi, 1974.

Willems, Edgar, El valor humano de la educación musical. Barcelona, Paidós, 1981.

Willems, Edgar, Las bases psicológicas de la educación musical. Buenos Aires, Eudeba, 1964a.

Willems, Edgar, El ritmo musical. Buenos Aires, Eudeba, 1964b.

Willems, Edgar, La preparación musical de los más pequeños. Buenos Aires, Eudeba, 1962.

Yepes, Antonio, Introducción al Método Coral Kodály. Buenos Aires, Barry, 1968.

Recibido: 11.04.2017

Aceptado: 01.09.2017 